\title{
LA INSERCIÓN LABORAL DE LOS EGRESADOS DEL SISTEMA UNIVERSITARIO CON PERFIL DE INVESTIGADOR EN MÉXICO. Un breve análisis de factores históricos y problemas institucionales académicos.
}

EMPLOYMENT FOR UNIVERSITY GRADUATES WITH RESEARCH PROFILE IN MEXICO. A brief analysis of historical factors and academic institutional problems.

\author{
Mtra. Mónica Maisterrena González \\ (Universidad de Guadalajara, México) \\ monica.maisterrena@gmail.com
}

Recibido: 23/8/2017

Aprobado: 4/1/2018

\begin{abstract}
RESUMEN
El ejercicio de la investigación como profesión ha evidenciado problemas estructurales, políticos e históricos en la consideración del investigador como fuente primaria para la generación de conocimiento y, ulteriormente, del cambio social. El presente estudio muestra un breve análisis sobre los factores que prescriben la condición laboral de los egresados del sistema universitario mexicano con miras a convertirse en investigadores. Además, se ofrece un panorama histórico-social que da cuenta de las diferencias económicas y perceptuales entre la investigación en ciencias sociales y humanidades con respecto a las ciencias exactas y médicas, el cual puede traducirse en propuestas tangibles para una mejora del empleo de los investigadores del primer grupo.
\end{abstract}

Palabras clave: Investigación, trabajo, movilidad, instituciones académicas, ciencias sociales.

\section{ABSTRACT}

The professionalization of researchers has placed in the spotlight structural, political and historical problems concerning research as a primary source for knowledge generation and, ultimately, of social change. This study presents a brief analysis of the factors that prescribe the job opportunities of graduates from the Mexican university system, who plan to become researchers. Moreover, the text shows a socio-historic landscape depicting the economic and perceptual differences between research in the social sciences/humanities and the engineering and medicine. These could translate into tangible proposals for job betterment of the former.

Keywords: Research positions, employment, mobility, academic institutions, social sciences.

\section{Introducción}

El presente ensayo busca contribuir al análisis del empleo y movilidad laboral en el sector de la investigación en México mediante una reflexión sobre los problemas que enfrentan los investigadores para incorporarse en el ámbito laboral y los espacios reducidos con los que cuentan. El campo laboral de los investigadores mexicanos ha estado ineludiblemente ligado a la movilidad, siendo su principal problemática la insuficiencia estructural de empleo (oportunidades de trabajo en esa área) y de recursos económicos para la creación de espacios orientados a ese sector en el país. Para abrir sus 
oportunidades de empleo, los investigadores han conjugado diferentes procesos de movilidad tanto nacional como internacional.

Cabe señalar que la eficiencia de un investigador depende de las bases formativas, estructurales y políticas, por tal motivo, en este ensayo se intentarán responder las siguientes preguntas: ¿la formación que reciben los universitarios o estudiantes de posgrado con perfil de investigador está contribuyendo para su incorporación laboral? ¿Cuáles son los factores que prescriben la condición laboral de los egresados universitarios con perfil de investigador? ¿Qué alternativas hay ante la subcontratación o el desempleo?

Para abordar el tema en cuestión se hablará del contexto laboral del investigador en el país desde una perspectiva histórico-social: el surgimiento, la institucionalización y la extensión de la investigación en México. Después se hablará del contexto actual de la investigación en distintos ámbitos y algunos de los componentes que influyen en la movilidad laboral de esa área.

\section{Perspectiva histórica de la investigación en México}

Para hablar del empleo del investigador en México es necesario comprender su historia, comprender cómo, para qué y dónde surge y se desarrolla. El investigador es el dispositivo central de la comunidad científica; su ejercicio está concentrado en una superficie institucional que lo avala, lo forma y le proporciona condiciones materiales, organizativas, teórico-metodológicas y cognitivas para realizar el trabajo de investigación.

A finales del siglo XVIII y al inicio del siglo XIX, la universidad surgió como principal sede institucional para la creación y reproducción del conocimiento (Maisterra, 2016: 6). Desde el planteamiento de Wallerstein (2011), en el siglo XIX las distintas disciplinas se abrieron para cubrir una gama de posiciones epistemológicas: por un lado, estaban las matemáticas (no experimentales), las ciencias naturales experimentales (física, química y biología); y, por otro lado, las humanidades (filosofía, artes y letras). Entre las humanidades y las ciencias naturales estaban la historia y las ciencias sociales. La primera era más cercana a las humanidades, y las segundas (nomotéticas) se inclinaban más a las ciencias naturales (Chavoya, 2002: 2). Indirecta o directamente "la institucionalización de la enseñanza fue acompañada de la institucionalización de la investigación” (Maisterra, 2017: 7).

En México fue hasta finales del siglo XIX con el proyecto de progreso del Porfiriato, que se dan los primeros acercamientos a la ciencia, tales como la fundación y organización del Observatorio Astronómico Nacional, la Biblioteca Nacional, la Dirección de Estudios Biológicos y la Comisión Geológica (Peña, 1995: s/p). A pesar de ello, en ese periodo no se tuvo la visión de la importancia de la ciencia para la mejora del país.

El trabajo de investigación de las diversas disciplinas se desarrolla, principalmente, en las universidades públicas. A partir de 1929 se incorporaron a la UNAM grupos de investigación como el Observatorio Astronómico Nacional, el Instituto de Biología y el Departamento de Exploraciones y Estudios Geológicos, con la Red de Sismología Nacional, bajo el nombre, los dos últimos, de Instituto de Geología. Años después, entre los treinta y los cincuenta, buscaban desarrollar otras ciencias, por lo que se crearon los institutos de Geografía y de Física, el Instituto de Matemáticas y de Geofísica, algunos institutos nacionales de Salud (Peña, 1995: s/p) y otros de Ciencias Sociales.

No obstante, la investigación era una actividad marginal, se contaba con un débil crecimiento institucional y poca demanda estudiantil y de académicos. La creación del Instituto de Investigaciones Sociales (IIS) en la UNAM en 1930, bajo la dirección de Alfonso Caso, fue uno de los principales espacios donde se construyó un programa de investigación integral en el que participaron distintas disciplinas. Para 1939, durante la dirección de Lucio Mendieta y Nuño en el IIS de la UNAM, este 
personaje organizó el instituto por áreas, entre las cuales se encontraba la Sociología, Medicina Social, Ingeniería y Arquitectura Social, Economía y Trabajo de Biblioteca, Archivo y Relaciones Exteriores.

A pesar de que se fueron abriendo paulatinamente algunos institutos de investigación en México, antes de los años cincuenta quienes estaban interesados en una carrera científica se iban al extranjero y ahí obtenían su doctorado. Muchos de ellos lograron consolidar grupos de trabajo en México una vez que regresaban y buscaban continuar con los proyectos que habían trabajado fuera, no obstante, esa tarea no fue fácil debido a que les hacía falta equipamiento para trabajar (Peña, 1995: s/p). En ese periodo, eran organismos no gubernamentales, como la Asociación Mexicana de Ciencias (AMC), entre otras, las que buscaban mejorar la enseñanza de la ciencia en el país (Villagómez, 2012) y crearon varias sociedades científicas como refugios o como clanes. Sin embargo, hacía falta contar con políticas públicas y apoyo del gobierno para fortalecer esa área. Además, se requería que la investigación científica se realizara en otras partes de la República Mexicana, ya que hasta los años setenta, gran parte de la investigación se concentraba en la UNAM.

En los años sesenta se fueron organizando y consolidando un número significativo de posgrados y se inicia una época de mayor actividad. Esto se debió a varios elementos: a) Pablo González Casanova aumentó los salarios de tiempo completo de la UNAM lo que hizo más atractiva la carrera académica; b) entre la década de los setenta y principios de los ochenta se crearon varios centros e institutos de investigación (tanto de ciencias exactas, naturales, sociales y humanidades), lo cual implicó la incorporación de nuevos investigadores ${ }^{1}$; c) se creó el Consejo Nacional de Ciencia y Tecnología (CONACYT) en 1971, con el cual se otorgaron becas para acelerar la formación de los nuevos investigadores y para que los estudiantes salieran al extranjero; d) se inició la descentralización de la investigación científica y se organizaron grupos en distintas ciudades del país. En términos generales, se contó con más recursos para lograr la expansión, los grupos más importantes eran los promovidos por la UNAM, CONACYT, CINVESTAV o la SEP (Peña, 1995: s/p). Tales políticas nacionales destinadas a crear y fortalecer grupos y proyectos de investigación en las universidades del país favorecieron el desarrollo de la investigación en ese periodo.

En el caso de las ciencias sociales, aunque algunos centros de investigación se crearon entre los años treinta y cuarenta -tales como el Instituto de Investigaciones Sociales (IIS) de la UNAM fundado en 1930, o bien, el Colegio de México en 1938-, la expansión de éstos fue tardía. Según Béjar y Hernández, el 83\% de los centros de investigación en ciencias sociales que existían hasta el año 2000, fueron creados después de 1970 (Chavoya, 2002:4), tal es el caso del Instituto de Educación Superior (IES) creado en 1975, el cual fue creado como un centro dedicado exclusivamente a la investigación ya que ésta escaseaba en todas las áreas. En Guadalajara, por ejemplo, a inicios de la década de los setenta surge el Centro de Investigaciones Socioeconómicas (CISE) en la Facultad de Economía de la Universidad de Guadalajara, donde inician las labores de investigación profesores de tiempo completo, que antes de la fundación de ese centro, se dedicaban a la docencia (Chavoya, 2002: 5). Es a partir de los años ochenta que comienza a expandirse la investigación científica como una función sustantiva importante en sí misma y cambian las condiciones para su desarrollo. Pues bien, conforme se distribuyen más recursos y se cuenta con más institutos, también incrementa la demanda y los trabajos para los investigadores.

La institucionalización es el proceso que conduce a que una actividad, como la investigación, sea aceptada y reconocida en la sociedad. En Guadalajara una de las instancias que favorecieron ese hecho fue la creación del Departamento de Investigación Científica y Superación Académica (DICSA), en 1983, el cual contaba con un apoyo financiero para el área de ciencias sociales. Debido a que el impulso a la investigación surgió "en las alturas de la burocracia universitaria de entonces” (Acosta, 2005: 7), eso permitió el crecimiento de los recursos institucionales dedicados a la investigación y el reconocimiento de la actividad científica a nivel local. No obstante, un año después de la creación del

\footnotetext{
${ }^{1}$ Egresados universitarios que iniciaban su trayectoria profesional como investigadores.
} 
DICSA, a nivel nacional se funda el Sistema Nacional de Investigadores (SNI), el cual permitió un incremento considerable de investigadores en el país.

La presencia de organismos como el IES, el DICSA y otras instituciones de esa índole brindó oportunidades de desarrollo profesional dentro del área de investigación a recién egresados de carreras como la sociología en la ciudad de Guadalajara ${ }^{2}$. Gracias a esos apoyos financieros, para los años noventa se formaron nuevos centros e instituciones de investigación predominantemente del área de ciencias sociales en la ciudad, paradójicamente en un periodo en el que estaba la crisis económica nacional y financiera de la universidad (Acosta, 2005: 7; Chavoya, 1998: 156; Chavoya, 2002: 9, Villagómez, 2012: s/p).

Mientras a nivel nacional el presupuesto destinado a la investigación era de 0.35\% entre 1990 y 1995 (Ibarra, 2010: 2), en Guadalajara se destinó poco más del 4\% en 1992 (Acosta, 2005: 7). De ahí que no sea de sorprender que en el DICSA, de contar con 15 investigadores en 1983 pasaron a ser 500 en 1994 (Chavoya, 2002: 10). Además, en ese lapso se formaron 27 dependencias de investigación en esa ciudad, lo que contrajo impacto en términos laborales, académicos y políticos en las estructuras universitarias. En lo que concierne a la Universidad de Guadalajara, los departamentos que anteriormente eran centros o institutos de investigación son los que contaron con mayor número de investigadores.

En el plano nacional, se observa que conforme van aumentando las plazas de investigación va creciendo también la producción. Entre más libertad se tenga para seleccionar temas de investigación hay más oportunidades de desarrollo de este sector, siempre y cuando se cuente con apoyos para su realización. Sin embargo, los apoyos recibidos por parte del gobierno para los investigadores han variado según las áreas y los temas de interés institucional. En el caso de Guadalajara, en las ciencias sociales de contar con más del 80\% del presupuesto destinado en 1985, pasó a ser el área con menor apoyo en 1990; mientras que en ciencias exactas e ingeniería de contar con el 9.2\% en 1985, pasaron al 52.8\% para 1990 (Chavoya, 2002: 16). Esta distribución no ha sido azarosa si se considera que la expansión de los centros de investigación y el apoyo para su desarrollo obedecen a múltiples factores, tales como: la importancia que va adquiriendo en la sociedad la dinámica disciplinaria (sus rasgos y características de la institución); así como las exigencias y demandas políticas y económicas para el progreso del país. Es decir, frente a un aparente deslumbramiento por el desarrollo científico y tecnológico, se suele privilegiar o priorizar el apoyo a investigaciones de esa índole y relegar otras áreas de estudio, como las ciencias sociales y humanidades, lo cual limita o privilegia el desarrollo profesional de los mismos.

\section{Tipos de empleos para los investigadores a partir de los años noventa}

En la década de los noventa, sectores gubernamentales e instituciones de educación superior comenzaron con programas de internacionalización a través de convenios, congresos internacionales y la participación en alianzas y redes académicas. En el caso del CONACYT, uno de los propósitos era organizar los perfiles de la planta nacional de investigadores conforme a estándares internacionales de formación profesional (Maisterra, 2016: 8-10).

En 1994 surgió una idea para que las universidades fortalecieran sus funciones de investigación y dejaran de ser universidades de docencia (Acosta, 2005: 4). Esto implicó, entre otras cosas: a) vincular la investigación con la docencia, cosa que ya ocurría en algunas áreas (tales como las ciencias sociales) y, b) el impulso a la investigación científica como mecanismo de transformación institucional y de fortalecimiento académico. Para ese impulso también se llevaron a cabo políticas de internacionalización de la ciencia: movilidad internacional de estudiantes e investigadores (Gacel, 2000). Con esa iniciativa se esperaba un avance significativo en el desarrollo de las disciplinas

\footnotetext{
${ }^{2}$ Entrevista a Juan Manuel Durán Juárez el 28 de octubre de 2015. Fue Coordinador de Investigación del Departamento de Investigaciones Científica y Superación Académica (DICSA) en 1986 y 1991.
} 
científicas y mayores empleos en investigación, sin embargo, en ese mismo periodo, las condiciones laborales se fueron deteriorando y comenzó a promoverse la proliferación de contratos laborales precarios.

A partir de este período comienza un declive en los espacios laborales para los recién egresados de carreras de ciencias sociales y humanidades. Además, surgen dificultades para adquirir un trabajo relacionado a la formación recibida (de investigación) y cambian también las condiciones de trabajo en cuanto a la seguridad laboral (prestaciones, antigüedad, seguro social, entre otras). El abanico de oportunidades laborales se reduce progresivamente.

¿A qué se deben tales condiciones de precariedad? Se pueden hablar de múltiples factores, sin embargo aquí me enfocaré en cuatro: 1) Aunque incrementaron los centros de investigación y, por ende, la planta académica y de investigadores (desligados de la docencia) en universidades públicas y otros institutos, las oportunidades de incorporación para las nuevas generaciones de investigadores se reducen debido, entre otras cosas, al problema de la jubilación de los investigadores predecesores; 2) a partir de 1989 el modelo neoliberal contrae cambios en el campo laboral. El empleo sufre profundas transformaciones, por ejemplo, se da la pérdida del empleo formal, se propugnaron leyes que sostenían modalidades de contratación más flexibles, y se redujeron las condiciones laborales formales (prestaciones, seguro, pensión, entre otros beneficios), lo cual afectó a distintos sectores, entre los cuales está el de la investigación, ya que muchos de ellos comienzan a ser subcontratados; 3) se da un aumento considerable de matrícula en las universidades debido a las nuevas exigencias laborales (en relación a la formación requerida), y eso dispara una demanda laboral mayor a la oferta de empleo existente; 4) Aunque la investigación normalmente se ha asociado con los estudios de posgrado, en algunas áreas, tales como las ciencias sociales, egresados de licenciatura podían incorporarse a la investigación y adquirir plazas. Sin embargo, en la actualidad, quienes egresan de las carreras de licenciatura requieren estudiar un posgrado para aspirar a ser investigadores y no asistentes (independientemente de que sea un contrato por tiempo indeterminado o eventual), sin tener una garantía de obtener el empleo deseado.

La relación entre investigación y posgrado es muy estrecha en la actualidad, principalmente porque éstos podrían ser la continuación de una formación como investigadores, "el acumulado de egresados de doctorado es la parte más importante del acervo de recursos humanos para la ciencia y la tecnología, pero representa una cantidad ínfima de la población económicamente activa” (Chavoya, 2013: 2), muchos de ellos están sin empleo por la falta de oferta de trabajo formal. Tan solo en el 2011, uno de cada tres egresados de posgrados obtenía un empleo (La Jornada, 2011). Existen distintas becas y apoyos para los estudiantes de posgrado a nivel maestría y doctorado, pero se ha descuidado el egreso y la estabilidad laboral de éstos. Otro problema es que algunos egresados de las distintas carreras ven como una opción laboral la inserción a un posgrado con acceso a becas, sin preocuparse realmente por adquirir habilidades y conocimiento; también están los casos de quienes sí aspiran a ser formados en investigación en algún posgrado, pero al no poder ingresar al sistema universitario como investigadores o poder ejercer, dirige éste fenómeno a un problema de sobre educación:

El creciente proceso de saturación habría implicado el aumento en importancia de profesionistas empleados en ocupaciones para las cuales no resultan necesarios los conocimientos y habilidades adquiridos en las aulas universitarias. Ello habría tenido consecuencias desfavorables sobre la determinación de los ingresos que perciben los profesionistas en el mercado laboral (Hernández, 2004: 99).

Ante ese fenómeno, se han diseñado programas posdoctorales nacionales; programas de retención en modalidad de incorporación de investigadores sin plaza a instituciones y centros de investigación, los cuales han contribuido en disminuir el desempleo en ese sector. Sin embargo, aunque existen esos programas u otros como PRODEP (antes PROMEP), CONACYT, entre otros, para el año 2012 "solamente ha podido tener empleo en una institución de educación superior una tercera parte de los egresados” (Chavoya, 2013: 5). 
El CONACYT, el PRODEP o el concurso de plazas vacantes en las diferentes IES son las oportunidades laborales más factibles para este sector, sin embargo, no es tarea fácil adquirir una plaza nueva, principalmente si se trata del área de ciencias sociales y humanidades. Un ejemplo de ello es que, aunque el trabajo de investigación se desarrolla principalmente en las universidades públicas, hay otras instituciones privadas que establecen compromiso con la investigación para impulsar la calidad académica, tal es el caso del Tecnológico de Monterrey, donde se produce conocimiento en relación al aspecto tecnológico (la nanotecnología, entre otros).

A nivel nacional, en el 2003 el 10\% de los miembros del SNI eran del área de Ciencias Médicas y de la Salud: 924 de ellos eran especialistas en neurociencias, infectología, inmunología, farmacología y salud pública (Ramírez, 2014: s/p). En el 2012 se incrementó a 1,906 investigadores. Según Julia Tagüeña Parga ${ }^{3}$, medicina es una de las ramas de la ciencia en que México se destaca a nivel internacional, además de los artículos mexicanos de astronomía y de física de altas energías (Informador, 2015).

No obstante, en México, los diferentes diagnósticos del sistema educativo nacional destacan la escasez de personal dedicado a la ciencia y a la tecnología. El CONACYT (2012a) considera que es necesario el incremento de los recursos capacitados para la investigación, sin embargo, para ello se busca "elevar la cantidad de recursos humanos calificados en ciencia y tecnología, y la vía es justamente el aumento de doctores graduados en las diferentes áreas y especialidades” (Chavoya, 2013: 2). Por su parte, en el caso de las ciencias sociales, en las instancias de investigación, se podría decir que el campo laboral se reduce a la academia, espacio en el que hay pocas posibilidades de incorporarse como recién egresados (aún si se trata de un posgrado) debido, entre otras cosas, a que las plazas están saturadas y quienes cuentan con ellas dentro de las instituciones aún no están en edad de jubilarse o bien, no desean hacerlo.

Por otra parte, además de la incorporación a las plazas de investigación que otorgan distintos organismos institucionales, los nuevos investigadores ${ }^{4}$ se enfrentan a un contexto laboral de subcontratación. Debido a que las plazas de investigación llegan a estar restringidas, muchos recién egresados de alguna licenciatura, maestría o doctorado reciben trabajos temporales enfocados a la investigación: principalmente proyectos gubernamentales, en consultorías o espacios donde se realizan estudios de mercado, por mencionar algunos. Por estas razones, muchos de ellos comienzan a ver como una opción la movilidad laboral.

\section{La movilidad laboral del investigador en México}

A mediados del siglo XX, personajes como Lucio Mendieta y Núñez, Pablo González Casanova, Efrén Carlos del Pozo, entre otros, impulsaron y procuraron los medios para que los investigadores de distintas áreas pudieran existir en México, algunos de ellos tuvieron la iniciativa de crear institutos de investigación, otros apoyaron con el aumento de plazas de tiempo completo y apoyo a los institutos ya existentes. Eso tuvo implicaciones en el atractivo de la carrera académica. No obstante, se contaba con presupuestos insuficientes, principalmente en lo que correspondía a los equipos y otros materiales. Ante ese panorama, muchos se vieron en la necesidad de buscar apoyos y establecer vínculos con fundaciones extranjeras para adquirir recursos económicos. Fue hasta finales de los setenta y principios de los noventa que México otorgó mayores apoyos financieros para esa área: se crearon más centros e institutos de investigación, hubo cada vez más investigadores, y se establecieron vínculos internacionales, ya no sólo para adquirir recursos, sino también para el desarrollo de la investigación en el país.

\footnotetext{
${ }^{3}$ Directora adjunta de Desarrollo Científico del Consejo Nacional de Ciencia y Tecnología (Conacyt)

${ }^{4}$ Recién egresados de carreras universitarias o posgrados con un perfil de investigadores, o bien, que comienzan una trayectoria laboral y profesional en esa área.
} 
El IES comenzó a establecer ligas con otros organismos relacionados con la investigación en ciencias sociales. En 1979, ingresó como miembro del Consejo Mexicano de Ciencias Sociales (COMECSO) y posteriormente se incorporó al Consejo Latinoamericano de Ciencias Sociales, con lo que adquirió proyección nacional e internacional (Chavoya, 2002: 7).

En América Latina, la globalización científica empezó a generar sus primeros efectos en los años noventa. En países como México la vinculación internacional comenzó con antelación, aunque fue a partir de los años noventa que se hizo más presente. En Guadalajara, por ejemplo, la expansión de la investigación se inició con la creación del Departamento de intercambio Académico en 1979, dependencia que se convirtió en la promotora del desarrollo académico de la Universidad de Guadalajara. Dentro de esa dependencia se creó una Coordinación de Apoyo a la Investigación a través de la cual se buscaron apoyos externos para el desarrollo de la investigación (Chavoya, 2002: 9). Con ello, los investigadores de las distintas disciplinas científicas han logrado atraer fuentes de financiamiento externo que auxilian las labores de investigación. Según sostiene Chavoya (2002:16), la mayoría de esos recursos provenían de organismos públicos tales como CONACYT, SEP, FOMES, entre otros.

Poco a poco fue incrementando la cantidad de investigadores inmersos en proyectos de investigación, algunos de envergadura internacional. Ese fenómeno de integración a grandes orbes científicos, ha beneficiado la productividad y el contacto científico de los investigadores mexicanos (Ibarra, 2010: 3), principalmente áreas como medicina, ciencias duras, naturales y tecnológicas o de ingeniería. Las ciencias básicas y las ciencias naturales presentan un índice mayor de cooperación internacional que aquél que presentan las ciencias aplicadas y las ciencias sociales (Maisterra, 2016: 11).

Aunque fueron creciendo paulatinamente los centros e institutos de investigación, y eso implicó recibir distintos apoyos, becas y recursos gubernamentales, extranjeros y privados, a partir de los años noventa las oportunidades laborales para los nuevos investigadores iban en detrimento, así como la competitividad a nivel internacional. De acuerdo al reporte 2008-2009, emitido por el World Economic Forum (Foro Económico Mundial), "México bajó del lugar 52 al 60 en competitividad a nivel mundial, quedando por debajo de países como Chile y Costa Rica” (Ibarra, 2010: 5).

Por otra parte, la inserción laboral para los egresados de posgrado no ha sido la más sencilla, ya que cuentan con pocas oportunidades para ejercer. La consultoría se vincula tanto a la gestión comercial, legal o financiera (y es uno de los servicios de subcontratación donde participan principalmente economistas, administradores, ingenieros y contadores), así como a la gestión social, en la que sociólogos, psicólogos y comunicólogos tienen una fuerte presencia (Beltrán y Strauss, 2011 citados por Blois, 2014: 112).

En cuanto a los apoyos que otorgan el PRODEP, el CONACYT, entre otros programas, son principalmente para estudiantes de posgrado o investigadores de planta, pertenecientes a un centro o instituto, no para recién egresados. En ese sentido, hay una sobreeducación universitaria que debe atenderse, más que con la reducción de la matrícula, con políticas que brinden oportunidades laborales orientadas a la formación profesional en distintas áreas e instancias.

Además de ese fenómeno, la subcontratación y el desempleo son aspectos que repercuten no sólo a los sectores más marginados sino también a estas áreas disciplinarias. No obstante, el problema de la precariedad laboral del egresado universitario, no sólo se relaciona con los trabajos temporales o el desempleo, sino también con aquellos oficios que poco se relacionan con la carrera o posgrado.

Ese tipo de condiciones laborales perecederas llevan, en este caso, a los recién egresados de carreras o posgrados enfocados a la investigación, a contar con trabajos eventuales (en el mejor de los casos). La movilidad laboral es un efecto de los trabajos temporales. Según Faret (2001:8) la movilidad es por definición una “deslocalización”, aparece también como un desplazamiento y una reconfiguración de los referentes de identificación del individuo (González, 2009: 64). Cebrián (1991 citado por García y Malo, 1996:106) relaciona la movilidad con la flexibilidad del mercado de trabajo. En ese sentido, la 
movilidad laboral es parte de las nuevas modalidades de empleo para los investigadores, ya que este concepto se entiende como los movimientos que realiza una persona en cuanto a los puestos de trabajo, de empresa, de región, de estado o de país.

La movilidad laboral puede explicarse desde la teoría de la "exclusión”, la cual dice que "los trabajadores informales son parte de un grupo en desventaja, que han sido excluidos de los beneficios otorgados por el Estado y de los circuitos de la economía moderna” (Perry et al., 2007 citado por Tornarolli y Conconi, 2007: 3). O bien, puede entenderse como un proceso de mejora laboral. Espejo (1996) menciona que la movilidad laboral hacia un mejor trabajo responde al logro de una categoría ocupacional superior a la de origen, o de la misma categoría ocupacional pero mejor recompensada (Chavoya, 2013: 7). En el caso del sector de la investigación, la movilidad internacional podría responder a esa última característica.

La vinculación internacional de la investigación se puede relacionar con las redes de movilidad, las cuales se han ido construyendo colectivamente a lo largo del tiempo. Tanto el SNI, el CONACYT, como otros organismos, han sido cruciales para la estandarización internacional del campo de la investigación en México. La movilidad internacional académica y estudiantil también juega un papel crucial en la formación, la actualización e inserción laboral de los investigadores. El reconocimiento académico y el liderazgo son elementos fundamentales porque permiten crear alianzas y proyectos entre academia, gobierno y organismos internacionales (Maisterra, 2016: 80).

A través de dichas prácticas, los investigadores buscan anclarse en una amplia gama de empleos o contar con mejores condiciones laborales. Esta estrategia de flexibilidad y desregularización laboral les coloca en condiciones de intercambio y les empuja a entrar y salir de diversos nichos económicos que involucran nuevas relaciones, otra distribución de tiempo y espacio.

\section{Conclusiones}

En el documento se observa un desequilibrio entre la demanda por parte de los sujetos que investigan de las distintas áreas, a la oferta recibida por parte de las instancias de investigación. Es decir, mientras que actualmente las ciencias exactas o ingenierías, así como las ciencias médicas y de la salud, reciben mayor apoyo económico e importancia por parte de las instancias de investigación (tales como CONACYT, SNI, entre otras), así como mayor reconocimiento social y político, en comparación con las ciencias sociales y humanidades, hay una escasez de personal en el plano de investigación de las áreas de ciencia y tecnología, y una sobre demanda o exceso de personal en las áreas de ciencias sociales.

La falta de recursos económicos para la investigación y el desarrollo científico repercute no sólo en términos estructurales, sino también individuales, en tanto que disminuye las oportunidades formativas y laborales de quienes aspiran dedicarse a la investigación en estas áreas que tienen un menor reconocimiento, además de que limitan las posibilidades de creación y producción. El crecimiento de áreas y grupos de investigación requiere reunir distintos componentes: que haya políticas estatales que incentiven el desarrollo de la investigación en las distintas áreas del conocimiento; que se promueva la productividad no sólo en lo que corresponde a la ciencia y la tecnología, sino también a las ciencias sociales y humanidades; que las instancias de investigación promuevan la productividad con calidad, es decir, que brinden las condiciones materiales, de tiempo e intelectuales para que se generen investigaciones novedosas y de calidad en las distintas áreas; que se abran nuevas plazas de investigación para que se incorporen nuevos investigadores; que los posgrados cumplan con su función formativa.

Por otra parte, en la actualidad, México no es un país sobresaliente en el desarrollo tecnológico a nivel internacional, sino que tiende a ser consumidor de las tecnologías producidas en otros países. Esta condición de cliente y no de productor lo sitúa en una posición de desventaja respecto a los recursos 
económicos que se otorgan para formar individuos y producir conocimiento en el área de ciencia y tecnología (Villagómez, 2012).

El gasto del Producto Interno Bruto (PIB) en relación a investigación y desarrollo pasó de $0.28 \%$ en 1990 a 0.35\% en 1995 (Ibarra, 2010: 2); para el 2012 se asigna el 0.4\% del PIB a la investigación científica (Villagómez, 2012; Ramírez, 2014); y, para el 2015, hay un incremento mínimo, de 0.03\%, es decir, equivale a $0.43 \%$ del PIB, quedando en uno de los tres países que menos invierten en esa área (Forbes, julio 17 de 2015). Esto a pesar de que la UNESCO recomienda a los países en desarrollo invertir el 1.5\% del PIB, es decir, casi cuatro veces más de lo que invierte México. Esto se convierte en un problema puesto que los recursos económicos posibilitan crear nuevos centros de investigación, mejorar las condiciones de la educación superior y los programas de posgrado, por mencionar algunos ejemplos.

\section{En definitiva, tal como lo menciona Chavoya:}

[...] el quehacer científico no se puede comprender fuera de las estructuras en donde se realiza. Para la creación de nichos científicos es necesaria la confluencia de múltiples factores, tales como un contexto favorable, la voluntad de las autoridades, fundamentada en la legitimidad de la ciencia, para destinar recursos humanos, materiales y económicos para el desarrollo de la legitimidad de la actividad científica, la presencia de líderes académicos, con tradición y experiencia, capaces de constituir y mantener equipos y la existencia de jóvenes dispuestos a iniciar la carrera científica (2002: 2).

El estado actual del modo de contratación laboral de los nuevos investigadores es precario, la probabilidad de inserción a una instancia de investigación para un recién egresado de un posgrado es escasa (más aún si se trata de una carrera). Principalmente si se trata del área de ciencias sociales, las condiciones de ejecución son menos favorables que en ciencias de otras áreas.

En este orden de ideas, se puede entender que se le otorga poco valor al conocimiento sobre el funcionamiento social en la actualidad. Hay un desconocimiento sobre áreas como la sociología o la antropología y su aplicación en el sector laboral fuera de la academia, así como su importancia en la sociedad actual. Asimismo, parece que la falta de recursos económicos y materiales en esas áreas puede estar relacionada con la falta de reconocimiento, quizás porque no están dentro de los estándares de lo que se considera “útil” en la actualidad.

Tal como lo plantea Ordine (2013) las humanidades, así como las ciencias sociales "no dan ningún beneficio, no producen ganancias, pero sirve para alimentar la mente, el espíritu y evitar la deshumanización de la humanidad”. Con esto sostengo que las ciencias sociales son áreas disciplinarias (así como las humanidades o las artes) que quizás nos conducen a tener mejores condiciones sociales y estructurales, sin estar subordinadas necesariamente al éxito económico. Como se trata de la sociedad, este tipo de estudios brindan preguntas, explicaciones, comprensiones, críticas, interpretaciones o descripciones de los fenómenos sociales. Es decir, aunque no se producen objetos tangibles (fuera de las publicaciones o libros) sí pueden producir propuestas o brindar herramientas para generar cambios y mejoras sociales.

Por tanto, es necesario mejorar las condiciones de investigación de todas las áreas, para que México logre, entre otras cosas, ser generador y no consumidor o maquilador de conocimiento. Ya que tanto en el área de ciencias sociales como en otras disciplinas científicas, no se cuenta con la formación y los recursos materiales y económicos suficientes para crear y producir. Las plazas con PRODEP y las estancias posdoctorales resultan insuficientes para un país que requiere ampliar su planta de investigadores con alta calificación (Chavoya, 2013: 13). Para ello es fundamental una formación de investigadores más completa: se deben confrontar las deficiencias científico-tecnológicas y materialesformativas que impidan su desarrollo. Para eso es necesaria la colaboración de la academia, las empresas y el gobierno, actores que hacen viable que se impulse el conocimiento mediante recursos económicos, y a su vez, que el conocimiento contribuya a una mejor economía del país. 
Los centros de investigación, los institutos y laboratorios, son algunos de los espacios en los cuales puede ejercer un investigador, sin embargo, tal como ha sido mencionado, aunque ha habido un crecimiento en este sector, sigue siendo un proceso lento. La falta de recursos es uno de los obstáculos más grandes para el desarrollo de la investigación en México y para la empleabilidad de los futuros aspirantes a ese campo de trabajo. Es necesario promover y apoyar la realización de maestrías y doctorados con base en la investigación, reducir la subcontratación y erradicar el desempleo. Una de las vías está también en mantener los vínculos internacionales "el progreso científico está relacionado con la capacidad de innovar y producir conocimiento científico dentro de un esquema competitivo internacional” (Ibarra, 2010: 2). La movilidad laboral no es un problema siempre y cuando ésta implique los intercambios intelectuales y escalar para mejorar las oportunidades de trabajo.

\section{Referencias}

ACOSTA, A. (2005). Departamentalización y contexto organizacional: la experiencia de la Universidad de Guadalajara. En Revista Electrónica de Investigación Educativa, 7(1), 1-18 [en línea] Recuperado en http://redie.uabc.mx/vol7no1/contenido-acosta.html

BLOIS, J. P. (2014). Mercado, ocupación pública y sociología en Argentina”. En Nómadas, 41, 115129. Universidad Central Bogotá: Colombia.

CHAVOYA PEÑA, M. L. (2002). La institucionalización de la investigación en ciencias sociales en la Universidad de Guadalajara. En Revista de la Educación Superior, 31(121) [en línea]. Recuperado en http://resu.anuies.mx/archives/revistas/Revista121_S1A1ES.pdf

----. (2013). Ser investigador: la zanahoria de los doctores recién egresados en México. Estudio de caso. En Diálogos sobre Educación, 4(6), 1-16.

EL INFORMADOR (2015, en línea). México destaca en investigación médica en el mundo: Conacyt. Recuperado de http://movil.informador.com.mx/tecnologia/2015/620236/7/mexico-destaca-eninvestigacion-medica-en-el-mundo-conacyt.htm

ESTRADA, F. J. (2008). Centenario del nacimiento del Dr. Efrén C. Del Pozo, potosino emérito. En Cuadernos Potosinos de Cultura Científica, 1(5), 59-74. Consultado el día 25 de marzo de 2017 en http://galia.fc.uaslp.mx/ uragani/cuadernos/cuaderno\%205.pdf

FORBES (julio 17 de 2015, en línea). México, entre los países que menos invierten en investigación. Recuperado de https://www.forbes.com.mx/mexico-entre-los-paises-que-menos-invierten-eninvestigacion/

GACEL, J. (2000). La internacionalización de las Universidades Mexicanas: políticas y estrategias institucionales. En Asociación Nacional de Universidades e Instituciones de Educación Superior. Ciudad de México: ANUIES.

GARCÍA ESPEJO, I. (1999). Formación en el trabajo y movilidad laboral. En Universidad de Oviedo, 59, 195-219.

GARCÍA SERRANO, C.; y MALO, M. A. (1996). Desajuste educativo y movilidad laboral en España. en Revista de Economía Aplicada, 4(11), 105-131. España: Universidad de Alcalá de Henares.

GONZÁLEZ CHÉVEZ, L. (2009). Movilidad laboral. Imposición estructural para la incorporación indígena a los mercados de trabajo en contextos globales. En Revista Migración y Desarrollo. Disponible en http://www.scielo.org.mx/pdf/myd/n13/n13a3.pdf

HERNÁNDEZ LAOS, E. (2004, en línea). Panorama del mercado laboral de profesionistas en México. Recuperado de http://www.ejournal.unam.mx/ecu/ecunam2/ecunam0208.pdf 
IBARRA ARIAS, J. J. A. (2010). La ciencia mexicana ante los desafíos de la globalización: innovación y competitividad para trascender. En Revista Ciencia. Disponible en http://www.revistaciencia.amc.edu.mx/online/6851.pdf

ISUNZA VIZUET, G.; y SORIANO CRUZ, V. (2008) "Mercado de trabajo y movilidad en la ciudad de México" (Artículos y Miscelánea), en Revista del Centro de Investigaciones Económicas, Administrativas y Sociales del Instituto Politécnico Nacional, núm. 11. Mundo Siglo XXI: México. Pp. 45-56.

LA JORNADA (2011, en línea) Sólo uno de cada tres egresados de posgrado logra empleo: AMC. Recuperado de http://www.jornada.unam.mx/2011/05/22/sociedad/036n1soc

MAISTERRA SIERRA, O. A. (2016) Las estrategias de internacionalización de la investigación en ciencias sociales: El caso de las élites científicas de la Zona Metropolitana de Guadalajara, Jalisco. [Tesis de Maestría en Investigación Educativa] Guadalajara.

ORDINE, N. (2013) La utilidad de lo inútil. Manifiesto. Barcelona: Acantilado.

TORNAROLLI, L. y CONCONI, A. (2007). Informalidad y Movilidad Laboral: Un Análisis Empírico para Argentina. En Centro de Estudios Distributivos, Laborales y Sociales, 59. Argentina: UNLP.

VILLAGÓMEZ OVIEDO, C. (2012). Ciencia, tecnología e investigación en México. En Revista interiográfico de la división de arquitectura, arte y diseño de la Universidad de Guanajuato Disponible en http://www.interiorgrafico.com/edicion/decima-segunda-edicion-septiembre2012/ciencia-tecnologia-e-investigacion-en-mexico

WALLERSTEIN, I. (2011). Abrir las ciencias sociales. México: Siglo XXI. 